IP/BBSR/96-29

hep-th/9604164

\title{
Compactifications of M-Theory to Two Dimensions
}

\author{
Alok Kumar*and Koushik Ray ${ }^{\dagger}$ \\ Institute of Physics, \\ Bhubaneswar 751 005, INDIA
}

June 18, 2021

\begin{abstract}
Compactifications of M-theory to two dimensional space-time on $\left(K 3 \times \mathbb{T}^{5}\right) / \mathbb{Z}_{2}$ and $\left(K 3 \times K 3 \times \mathbb{S}^{1}\right) / \mathbb{Z}_{2}$ orientifolds are presented. These orientifolds provide examples of anomaly free chiral supergravity models in two dimensions with $(8$, $0)$ and $(4,0)$ supersymmetries. Anomaly free spectra at the enhanced symmetry points are also obtained. The results confirm the twisted sector contribution to the spectrum in the case of $\mathbb{T}^{9} / \mathbb{Z}_{2}$ discussed earlier.
\end{abstract}

\section{Introduction}

Various compactifications of M-Theory [1] 16] have been discussed recently in order to understand the duality symmetries of string theory in a unified framework. In particular a great deal of attention has been paid to the ten [4] and six dimensional [5, 6, 9, 10, 14] cases due to the nontrivial anomaly cancellation requirements in these dimensions. Since the full content of M-Theory is not known, the anomaly cancellation [17, 18] provides useful informations about its consistent compactifications. In particular this can be used to predict the contribution of the twisted sectors of these theories. For example, in the case of M-Theory on the orientifold $\mathbb{S}^{1} / \mathbb{Z}_{2} \mathbb{4}$, one gets the $E_{8}$ gauge multiplets from each of the two twisted sectors of the theory which gives the $E_{8} \times E_{8}$ heterotic string in ten dimensions. In the six dimensional case, one encounters a number of possibilities. In this case chiral supergravity theories can be constructed either with $N=1$ or $N=2$ supersymmetries. For the $N=2$ case the anomaly cancels for one gravity and 21 tensor multiplets. An explicit construction

*kumar@iopb.ernet.in

${ }^{\dagger}$ koushik@iopb.ernet.in 
of this type can be obtained from $M$-Theory when it is compactified on a $\mathbb{T}^{5} / \mathbb{Z}_{2}$ orientifold. In the $N=1$ case, there are several ways that this restriction is satisfied. One such possibility is for one gravity, 9 tensor, $m U(1)$ vectors and $(m+12)$ hypermultiplets [18]. These are also anomaly free when the symmetry enhances at special points of the moduli space in these theories in certain directions [9]. An explicit example for a model of this type for $m=0$ is obtained by type IIB string compactification on an orientifold $K 3 / \mathbb{Z}_{2}\left[19,20\right.$. $M$-Theory compactification on $K 3 \times \mathbb{S}^{1} / \mathbb{Z}_{2}$ [9, 10] and their further projections give many such models with different number of vector multiplets.

Another case, where the anomaly cancellation puts nontrivial restrictions on the construction of consistent theories is two-dimensions. The anomaly contributions of the spin- $\frac{1}{2}$ and spin- $\frac{3}{2}$ Weyl spinors in two dimensions are respectively [17],

$$
I_{\frac{1}{2}}=-\frac{1}{24} p, \quad I_{\frac{3}{2}}=\frac{23}{24} p,
$$

where $p$ is the anomaly polynomial. In addition, a chiral right-moving scalar also contributes to anomaly in two dimensions which is equal to that of the positive chirality spin- $\frac{1}{2}$ Weyl field. Examples of anomaly cancellations appear in the context of the first quantized string constructions. For known string theories with $(p, q)$ supersymmetries, the anomaly trivially cancels when $p=q$. For $p \neq q$, it is well known that the gravitational anomaly on the string worldsheet cancels for the field content of the heterotic string. In this case, the anomaly cancellation gives the difference between the numbers of left and right-moving fermionic coordinates $(=22)$ in the heterotic string theory in its fermionic formulation.

Anomaly cancellation in two dimensions in the context of $M$-Theory compactification were discussed in [5] where construction of these theories on $\mathbb{T}^{9} / \mathbb{Z}_{2}$ orientifolds have been discussed. A new feature of the $\mathbb{T}^{n} / \mathbb{Z}_{2}$ orientifold was noticed in [5], where it was argued that the fixed points of the discrete group are not uniformly distributed along the sides of the torus, implying a compactification on degenerate torii. It was conjectured that the only exception was the two dimensional case where all the 512 Majorana-Weyl (mw) fermions necessary for anomaly cancellation arise from 512 twisted sectors of $\mathbb{T}^{9} / \mathbb{Z}_{2}$ [5, 6]. This provides an example of $(16,0)$ supergravity model in two dimensions.

In view of the significance of $M$-Theory in understanding the duality symmetries and other aspects of string theories, it is important to analyze other compactifications of this theory. In this paper, we discuss other orientifold compactifications to two dimensions, namely $\left(K 3 \times \mathbb{T}^{5}\right) / \mathbb{Z}_{2}$ and $\left(K 3 \times K 3 \times \mathbb{S}^{1}\right) / \mathbb{Z}_{2}$. By defining the $\mathbb{Z}_{2}$ symmetry on the eleven-dimensional fields as well as on the compactified coordinates, including the harmonic forms on $K 3$, we explicitly compute the fermionic and bosonic zero modes for these cases. Then by combining these with the twisted sector states, namely 256 and $128 \mathrm{mw}$ fermions in the two cases, the anomaly cancellation condition is satisfied. Our results confirm the contribution of the twisted sector states mentioned 
above for $\mathbb{T}^{9} / \mathbb{Z}_{2}$ in [5, 6]. We also study the enhanced symmetry points in these cases using the equivalence of $M$-Theory on $K 3 \times \mathbb{S}^{1}$ with the heterotic string on $\mathbb{T}^{4}$. We find the particle spectrum at these points and once again show the anomaly cancellation.

\section{$2 \quad \mathbb{T}^{9} / \mathbb{Z}_{2}$ orientifold}

We now begin by reviewing the case of $M$-Theory on $\mathbb{T}^{9} / \mathbb{Z}_{2}$ and apply the techniques to the $\left(K 3 \times \mathbb{T}^{5}\right) / \mathbb{Z}_{2}$ and $\left(K 3 \times K 3 \times \mathbb{S}^{1}\right) / \mathbb{Z}_{2}$ orientifolds subsequently. We shall find both the bosonic and the fermionic spectrum for the $\mathbb{T}^{9} / \mathbb{Z}_{2}$ orientifold. They coincide with the one mentioned in [5, 6] on the basis of supersymmetry multiplet structure. First, let us present the spectrum for compactification on $\mathbb{T}^{9}$. Then by defining the $\mathbb{Z}_{2}$ projection we obtain the spectrum for $\mathbb{T}^{9} / \mathbb{Z}_{2}$. Since the only bosonic fields that enter into the counting of the physical degrees of freedom are the graviton and the scalars, we restrict to these fields only. For toroidal compactification, the bosonic spectrum in two dimensions consists of $G_{\mu \nu}$ and $G_{a b}$ components of the the elevendimensional field $G_{M N}$. These give one graviton and 45 scalars in two dimensions. Only two dimensional fields originating from the eleven dimensional fields $A_{M N P}$ in two dimensions are the 84 scalars $A_{a b c}$. Together we have 1 graviton and 129 scalars. Since a graviton in two dimensions has $(-1)$ physical degree of freedom due to gauge fixings [5], they have to be taken into account in counting the number of scalars. Effectively only bosonic degrees of freedom are that of 128 scalars.

The fermionic degrees of freedom can be counted by decomposing the eleven dimensional Majorana spin- $\frac{3}{2}$ spinor into a set of spin- $\frac{1}{2}$ and spin- $\frac{3}{2}$ spinors in two dimensions. We find it more convenient to decompose the eleven dimensional Majorana spinor first in terms of the ten dimensional mw spin- $\frac{3}{2}$ fields $\Psi_{\bar{\mu}}^{ \pm}$and and spin- $\frac{1}{2}$ fields $\Psi_{10}^{ \pm}$. The two dimensional field content is then found by decomposing these as a product of the $2 \mathrm{~d}$ space-time spinor and an internal eight-dimensional spinor. $\Psi_{\bar{\mu}}^{+}$can be decomposed into a set of two dimensional spin- $\frac{3}{2}$ fields $\psi_{\mu}^{+} \chi_{8}^{+}, \psi_{\mu}^{-} \chi_{8}^{-}$and spin- $\frac{1}{2}$ fields $\psi^{+} \chi_{8 i}^{+}, \psi^{-} \chi_{8 i}^{-}, \mu=0,1$ and $i=1 . .8$, where $\chi_{8}^{ \pm}\left(\chi_{8 i}^{ \pm}\right)$are the spin- $\frac{1}{2}\left(\frac{3}{2}\right)$ modes on $\mathbb{T}^{8}$. As a result $\Psi_{\bar{\mu}}^{+}$yields 8 gravitinos $\left(\psi_{\mu}^{+}\right)$of positive chirality and 8 gravitinos $\left(\psi_{\mu}^{-}\right)$of negative chirality in two dimensions. We also have $64 \mathrm{mw}$ spinors $\left(\psi^{+}\right)$of positive chirality and 64 of negative chirality in two dimensions. Similarly $\Psi_{\bar{\mu}}^{-}$contributes 8 two-dimensional gravitinos of positive and 8 of negative chirality and gives $64 \mathrm{mw}$ spinors of positive and 64 of negative chirality. The ten dimensional mw spinor $\Psi_{10}^{+}$can be decomposed as a product $\psi^{+} \chi_{8}^{+}$and $\psi^{-} \chi_{8}^{-}$. It gives $8 \mathrm{mw}$ spinors of positive and 8 of negative chirality. The ten dimensional mw spinor $\Psi_{10}^{-}$ can also be decomposed into 8 positive and 8 negative chirality two dimensional mw spinors. To count the physical degrees of freedom, we note that each of the gravitinos in two dimensions has a $(-1)$ degrees of freedom [5] and cancels the physical degree of freedom of a mw spinor of opposite chirality. As a result the physical degrees of freedom in two dimensions consists of $128 \mathrm{mw}$ spinors of positive chirality and 128 of 
negative chirality. Combining them with the 128 scalars they form 8 scalar multiplets of $(16,16)$ supersymmetry in two dimensions. This is the maximal supersymmetry that can be obtained in two dimensions through compactifications of known higher dimensional theories. An infinite dimensional symmetry structure for these theories has been demonstrated and their integrability has been argued [21]. The coset space structure for this theory is known to be $E_{8(8)} / S O(16)$ which is parameterized by 128 scalars found above.

We next discuss the model which is obtained by an orientifolding of the one discussed above. The orientifolding is defined as a $\mathbb{Z}_{2}$ projection which acts on the eleven-dimensional 3 -form field by a change of sign, viz. $A_{M N P} \rightarrow-A_{M N P}$. At the same time the 11-dimensional gravitino transforms as $\psi_{M} \rightarrow \gamma^{11} \psi_{M}$. Here $\gamma^{11}$ is the analogue of the four-dimensional $\gamma^{5}$ and is defined as the product of tendimensional gamma matrices, $\gamma^{11} \equiv \gamma^{0} \cdots \gamma^{9}$. For the ten dimensional fields it implies, $\Psi_{\bar{\mu}}^{ \pm} \rightarrow \pm \Psi_{\bar{\mu}}^{ \pm}, \Psi_{10}^{ \pm} \rightarrow \mp \Psi_{10}^{ \pm}$. The $\mathbb{Z}_{2}$ symmetry also acts on the internal coordinates as $\left(x^{2}, \ldots, x^{10}\right) \rightarrow-\left(x^{2}, \ldots, x^{10}\right)$. The result of this operation on the fermionic coordinates can be obtained in various ways. A simple way to see their effect on the fermions is to notice that, in order to keep the worldsheet supercurrent single valued, the worldsheet fermionic coordinates are also odd under the above symmetry. Then by writing down the vertex operators for the spinors in terms of the bosonized fields $y_{1}, . ., y_{4}$ one finds, $\chi_{8}^{ \pm} \rightarrow \chi_{8}^{ \pm}$and $\chi_{8 i}^{ \pm} \rightarrow \mp \chi_{8 i}^{ \pm}$.

States in the untwisted sector consists of those left invariant under this $\mathbb{Z}_{2}$. All the 129 scalar fields as well as the graviton survive the $\mathbb{Z}_{2}$ projection. Among the fermions, the set of fields invariant under $\mathbb{Z}_{2}$ are $16 \mathrm{mw}$ spin- $\frac{3}{2}$ states with positive chirality and $144 \mathrm{mw}$ spin- $\frac{1}{2}$ states of negative chirality. 16 of these spin- $\frac{1}{2}$ states cancel the negative physical degrees of gravitinos. The presence of 16 gravitinos of positive chirality implies that the result of orientifolding of $\mathbb{T}^{9}$ compactification gives a $(16,0)$ local supersymmetric model in two dimensions. As expected, the number of supersymmetries reduces by a factor of half with respect to the compactification $\mathbb{T}^{9}$.

The total anomaly of the 144 negative chirality spin- $\frac{1}{2}$ and 16 positive chirality spin- $\frac{3}{2}$ is given by

$$
I=-144 I_{\frac{1}{2}}+16 I_{\frac{3}{2}}=\frac{512}{24} p .
$$

The anomaly is cancelled by $512 \mathrm{mw}$ spin- $\frac{1}{2}$ fields of positive chirality. On the basis of this fact, it was argued in [5, 6] that the twisted sectors contribute $512 \mathrm{mw}$ spinors, each one contributing one such fermion. It has also been pointed out [6] that these fermions are singlets under the $(16,0)$ supersymmetry mentioned above. As a result, the contribution of each of the twisted sector still comes as a supersymmetry multiplet and the 512 extra scalars mentioned in [5] are not needed for maintaining the supersymmetry structure.

It is also known [22] that a truncation of the $d=3, N=16$ supergravity leads to a $(16,0)$ supersymmetric model in two dimensions with the coset structure of the 
moduli fields being $E_{8(8)} / S O(16)$. This result coincides with the number of scalars found above.

\section{$3 \quad$ Orientifold of $K 3 \times \mathbb{T}^{5}$}

We now describe the compactification of $M$-theory on $K 3 \times \mathbb{T}^{5}$ and obtain its orientifold by a $\mathbb{Z}_{2}$ projection and by adding the twisted sector states. As in the case of $\mathbb{T}^{9}$, we shall ignore the one and higher-form fields in the bosonic spectrum. The necessary informations needed to obtain the bosonic sector of the compactified theory are the moduli space dimension of the Einstein metric on $K 3$, namely 58 . In addition one also needs the information that the only nonzero betti numbers for $K 3$ are $b_{0}=b_{4}=1$ and $b_{2}=22$. Out of the 22 two-forms on $K 3$, there are 3 self-dual and 19 anti-self-dual 2-forms. In addition we also use the fact that $K 3$ does not have any continuous isometries. From the eleven-dimensional graviton $G_{M N}$ one now obtains, in two dimensions, one graviton $G_{\mu \nu}$ and 58 scalars $G_{m n}$, where $m$ is symbolically representing $K 3$. In addition, one also has 15 scalars $G_{a b}$, where $a, b$ etc. are the indices on $\mathbb{T}^{5}$. By compactifying the eleven-dimensional three form $A_{M N P}$, one obtains, in two dimensions, 110 scalars $A_{m n a}$ and 10 scalars $A_{a b c}$. Combining these results we have a graviton and 193 scalars. As stated earlier, in two dimensions, one of the scalars compensates for the negative degree of freedom of the graviton and one is finally left with the 192 physical scalar degrees of freedom.

We will now find the fermionic degrees of freedom for this case. For this computation one needs the the index of the spin- $\frac{1}{2}$ Dirac operator and spin- $\frac{3}{2}$ Rarita-Schwinger operator on $K 3$ [23]. The spin- $\frac{1}{2}$ Dirac index on $K 3$ is given by

$$
I_{\frac{1}{2}}^{-}-I_{\frac{1}{2}}^{+}=2
$$

and the spin- $\frac{3}{2}$ index is given as

$$
I_{\frac{3}{2}}^{-}-I_{\frac{3}{2}}^{+}=-40 \text {. }
$$

The two dimensional fermionic spectrum is now obtained in the same manner as in the last section by decomposing the ten dimensional mw fermions $\Psi_{\bar{\mu}}^{ \pm}$and $\Psi_{10}^{ \pm}$ into a product of a two dimensional spinor, a spinor on $K 3$ and another one on $\mathbb{T}^{4}$. We denote the spin- $\frac{3}{2}\left(\frac{1}{2}\right) \mathrm{mw}$ fields in the two dimensional space-time by $\psi_{\mu}^{ \pm}$ $\left(\psi^{ \pm}\right)$; on $K 3$ by $\chi_{k m}^{ \pm}\left(\chi_{k}^{ \pm}\right)$and on $\mathbb{T}^{4}$ by $\chi_{4 i}^{ \pm}\left(\chi^{ \pm}\right)$. Then from $\Psi_{\bar{\mu}}^{ \pm}$we obtain four gravitinos $\left(\psi_{\mu}^{-} \chi_{K}^{-} \chi_{4}^{+}\right)$of negative chirality and four gravitinos $\left(\psi_{\mu}^{+} \chi_{k}^{-} \chi_{4}^{-}\right)$of positive chirality using the index of the spin- $\frac{1}{2}$ fields on $K 3$. One finds 80 spinors of positive chirality and 80 of negative chirality as $\psi^{+} \chi_{k m}^{+} \chi_{4}^{+}$and $\psi^{-} \chi_{k m}^{+} \chi_{4}^{-}$respectively from contributions of the spin- $\frac{3}{2}$ index on $K 3$. We also get 16 spinors of negative and 16 of positive chirality as $\psi^{-} \chi_{k}^{-} \chi_{4 i}^{+}$and $\psi^{+} \chi_{k}^{-} \chi_{4 i}^{-}$. Similarly the other mw spin- $\frac{3}{2}$ field in ten dimensions, namely $\Psi_{\bar{\mu}}^{-}$gives 4 positive and 4 negative chirality mw gravitinos in two dimensions. Once again we get 80 positive and 80 negative chirality spin- $\frac{1}{2}$ fields 
as contribution of the spin- $\frac{3}{2}$ index on $K 3$ and the index of the spin- $\frac{1}{2}$ fields on $K 3$ implies that there are 16 spinors of both chiralities. Using the spin- $\frac{1}{2}$ index on $K 3$, one can also decompose the ten dimensional spinors $\Psi_{10}^{+}$and $\Psi_{10}^{-}$. In each case one obtains 4 positive and 4 negative chirality spinors in two dimensions.

Thus we obtain, in two dimensions, a theory with eight positive and eight negative chirality spin- $\frac{3}{2}$ fields. Therefore we have an $(8,8)$ supersymmetric model in two dimensions. This model can also be obtained by a toroidal compactification of the heterotic string theory to two dimensions. This also follows from the fact that the $M$-Theory compactified to six dimensions on $K 3 \times \mathbb{S}^{1}$ is the dual description of the heterotic string theory compactified on $\mathbb{T}^{4}$. In fact, the number of scalars in two dimensions, viz. 192, is the same as that for the heterotic string theory on $\mathbb{T}^{8}$. The moduli space in this case is parameterized by $O(8,24) / O(8) \times O(24)$. Among spinor degrees of freedom, 8 positive and 8 negative chirality ones are used to cancel the negative degrees of the gravitino modes. One is finally left with 192 positive and 192 negative chirality spin- $\frac{1}{2}$ fields and all of them combine into 24 multiplets of the $(8,8)$ supersymmetry algebra in two dimensions.

After discussing the compactification on $K 3 \times \mathbb{T}^{5}$, we now obtain its orientifold through a $\mathbb{Z}_{2}$ projection and by adding appropriate twisted sectors. The $\mathbb{Z}_{2}$ action on the ten dimensional field was already defined in section-2, namely $A_{M N P} \rightarrow-A_{M N P}$ and $\psi_{M} \rightarrow \gamma^{11} \psi_{M}$. It acts on $\mathbb{T}^{5}$ as $x^{a} \equiv\left(x^{6}, \cdots, x^{10}\right) \rightarrow-\left(x^{6}, \cdots, x^{10}\right)$. This has the effect of transforming the spinors on $\mathbb{T}^{4}$, namely the internal part of the ten-dimensional mw spinor discussed earlier, as $\chi_{4}^{ \pm} \rightarrow \mp \chi_{4}^{ \pm}$and $\chi_{4 i}^{ \pm} \rightarrow \pm \chi_{4 i}^{ \pm}$.

The action of $\mathbb{Z}_{2}$ on the bosonic modes of $K 3$ is the same as the one discussed in 9, 10,24]. Under this symmetry, 34 of the $58 K 3$ moduli fields are even and 24 are odd. It also leaves 14 two-forms, including all the three self-dual two-forms invariant. Remaining 8 two-forms are odd whereas zero and four-forms are even. To describe the $\mathbb{Z}_{2}$ operation on the fermionic zero modes, one exploits their relations to the harmonic forms on the compact space. For example, the number of spin- $\frac{1}{2}$ modes equals that of the $(0, p)$ forms and the number of spin- $\frac{3}{2}$ modes equals twice the number of the $(1,1)$ forms [23]. As a result, all the spin- $\frac{1}{2}$ modes on $K 3$ coming from the $(0,0)$ - and $(0,2)$ - forms are invariant under the $\mathbb{Z}_{2}$ symmetry. On the other hand, only 24 of the 40 spin- $\frac{1}{2}$ modes coming from the 12 invariant (1,1)-forms are even under $\mathbb{Z}_{2}$ with 16 being odd. Using these informations, one can compute the number of bosonic and fermionic modes which survive the $\mathbb{Z}_{2}$ projection. One finds that the graviton $G_{\mu \nu}$ is invariant under this symmetry. Similarly 34 of the $K 3$ moduli $\left(G_{m n}\right)$ and 15 scalar modes on $\mathbb{T}^{5}$, namely $G_{a b}$ and $G_{10,10}$, are invariant. The components $A_{m n a}$ and $A_{a b c}$ contribute 70 and 10 scalar fields in two dimensions respectively. By cancelling one of the scalars with the negative degree of freedom of the graviton we are left with 128 scalars in two dimensions.

The $\mathbb{Z}_{2}$ operation on the spinors leaves the following set of two-dimensional fields invariant. From the mw spinor component $\Psi_{\bar{\mu}}^{+}$we get the following set of invariant fields, (i) 4 positive chirality spin- $\frac{3}{2}$ components $\left(\psi_{\mu}^{+} \chi_{k}^{-} \chi_{4}^{-}\right)$coming, e.g. from the 2 
(0, p) forms on $K 3$ and 2 surviving Killing spinors on $\mathbb{T}^{5}$, (ii) 16 spin- $\frac{1}{2}$ components $\left(\psi^{-} \chi_{k}^{-} \chi_{4 i}^{+}\right)$, (iii) 32 spin- $\frac{1}{2}$ positive chirality components $\left(\psi^{+} \chi_{k m}^{+} \chi_{4}^{+}\right)$, and (iv) 48 negative chirality spin- $\frac{1}{2}$ components $\left(\psi^{-} \chi_{k m}^{+} \chi_{4}^{-}\right)$. Similarly from $\Psi_{\bar{\mu}}^{-}$we get (i) 4 positive chirality spin- $\frac{3}{2}$ fields $\left(\psi_{\mu}^{+} \chi_{k}^{-} \chi_{4}^{+}\right)$, (ii) 32 positive chirality spin- $\frac{1}{2}$ fields $\left(\psi^{+} \chi_{k m}^{+} \chi_{4}^{-}\right)$, (iii) 16 negative chirality spin- $\frac{1}{2}$ fields $\left(\psi^{-} \chi_{k}^{-} \chi_{4 i}^{-}\right)$and (iv) 48 negative chirality spin- $\frac{1}{2}$ fields $\left(\psi^{-} \chi_{k m}^{+} \chi_{4}^{+}\right)$. The ten-dimensional fields $\Psi_{10}^{ \pm}$have the surviving components in two dimensions as $\psi^{-} \chi_{k}^{-} \chi_{4}^{+}$and $\psi^{-} \chi_{k}^{-} \chi_{4}^{-}$respectively. As a result, we have an $(8,0)$ chiral supergravity model in two dimensions coupled to a set of matter multiplets, i.e.

- $\left(1 g_{\mu \nu}, 1 \phi, 8 \psi_{\mu}^{+}, 8 \psi^{-}\right)$

- $\left(128 \phi_{L}, 128 \psi^{-}\right)$

- $\left(128 \phi_{R}, 64 \psi^{+}\right)$.

The presence of 64 positive chirality fermions is consistent with the $(8,0)$ supersymmetry as it has been pointed out earlier, in another context [25], that the chiral fermions on the worldsheet can be coupled to the $(8,0)$ supergravity theory. The situation is similar to the case of the heterotic string theory where there are extra worldsheet mw fermions of a particular chirality.

We have a surplus of $64 \mathrm{mw}$ fermions with negative chirality. A computation of anomaly contributions of different fields implies that it can be cancelled by 256 positive-chirality fermions from the twisted sectors of the theory. Since $K 3 \times \mathbb{T}^{5}$ have exactly 256 twisted sectors and the contribution of the individual twisted sector remains unchanged with respect to the compactification on $\mathbb{T}^{9} / \mathbb{Z}_{2}$ as in six dimensions [9], we get precise anomaly cancellation in this model with $(8,0)$ supersymmetry. Once again the twisted sector fermions are invariant under this supersymmetry. Next we analyze the case of enhanced symmetry and show that the theory is anomaly free in this case as well.

To show that the anomaly cancels at the enhanced symmetry point also, we follow the approach of ref. [9] in the six dimensional case. Symmetry enhancement takes place at points in the moduli space where there are extra massless multiplets. It has been argued in [9] that such multiplets, considered below, do not receive any quantum correction to their masses. The enhanced symmetry in the $M$-Theory compactification can be understood in terms of the strong-weak duality between the type IIA string theory on $K 3$ and the heterotic string theory on $\mathbb{T}^{4}$. All the states of the $M$-Theory on $K 3 \times \mathbb{T}^{5}$ are identified in terms of the heterotic string spectrum on $\mathbb{T}^{8} . \mathbb{Z}_{2}$ operations are also identified in the two cases. Then the $\mathbb{Z}_{2}$-projection in the heterotic string gives the enhanced symmetry for the $M$-Theory case. We also exploit the fact that the twisted sector spectrum does not change at the enhanced symmetry point [9].

To write down the states in the heterotic string theory, we denote the spacetime coordinates in the left-moving sector as $\left(X^{L \mu}, \psi^{L \mu}\right)$ and the internal eight- 
dimensional coordinates as $\left(X^{L i}, \psi^{L i}\right)$. The right-moving coordinates are denoted as $X^{R \mu}, X^{R i}$ and $X^{R I}$, where $X^{R I}$ are the sixteen extra internal coordinates in the right-moving sector. The momentum lattice in the internal eight-dimensional space is denoted by a sixteen dimensional momentum vector $\vec{k}$. Similarly the $E_{8} \times E_{8}$ root vectors are denoted by $\overrightarrow{k_{1}}$ and $\overrightarrow{k_{2}}$ respectively. Then the states of the $E_{8} \times E_{8}$ heterotic string theory, when it is compactified to two dimensions, are given as: (i) $\psi_{-\frac{1}{2}}^{L \mu}\left|0>\otimes X_{-1}^{R \nu}\right| \overrightarrow{0}, \overrightarrow{0}, \overrightarrow{0}>$, where the vacuum in the left-moving sector is denoted by $\mid 0>$ and in the right-moving sector by $\mid \overrightarrow{0}, \overrightarrow{0}, \overrightarrow{0}>$, with the entries denoting the momenta along the internal 16-dimensional lattice and two $E_{8}$ directions respectively. (ii) Scalar states: (a) $\psi_{-\frac{1}{2}}^{L i}\left|0>\otimes X_{-1}^{R j}\right| \overrightarrow{0}, \overrightarrow{0}, \overrightarrow{0}>$, (b) $\psi_{-\frac{1}{2}}^{L i}\left|0>\otimes X_{-1}^{R I}\right| \overrightarrow{0}, \overrightarrow{0}, \overrightarrow{0}>$, (c) $\psi_{-\frac{1}{2}}^{L i}|0>\otimes| \overrightarrow{0}, \vec{\alpha}, \overrightarrow{0}>$, and (d) $\psi_{-\frac{1}{2}}^{L i}|0>\otimes| \overrightarrow{0}, \overrightarrow{0}, \vec{\alpha}>$, where $\vec{\alpha}$ is a root vector of $E_{8}$. As a result we have 8 scalars in the adjoint representation of $E_{8} \times E_{8}$. In addition we also have 64 scalars which are singlets of $E_{8} \times E_{8}$. At a generic point in the moduli space, we have only 192 scalars, namely (ii.a) and (ii.b). Together they give the coset $O(8,24) / O(8) \times O(24)$ of the moduli fields. Note that the state (i) includes the dilaton. As a result we did not need to cancel any negative degree of freedom in this case. Same is true for the counting of fermions as well.

Fermions at the enhanced symmetry points can also be counted in exactly the same manner. The fermion states of the heterotic string theory in two dimensions are: $(i)^{\prime}\left|\bar{\psi}>\otimes X_{-1}^{\nu}\right| \overrightarrow{0}, \overrightarrow{0}, \overrightarrow{0}>$, where $\mid \bar{\psi}>$ is the left-moving spinor vacuum in the NSR formulation of the heterotic string theory. These are the 16 two-dimensional gravitino states, 8 with positive and 8 with negative chirality. $(i i)^{\prime} 64$ spinor states of positive and 64 of negative chirality: $\left|\bar{\psi}>\otimes X_{-1}^{i}\right| \overrightarrow{0}, \overrightarrow{0}, \overrightarrow{0}>$. (iii $)^{\prime} 8$ positive and 8 negative chirality spinors in the adjoint representation of $E_{8} \times E_{8}$ : (a) $\left|\bar{\psi}>\otimes X_{-1}^{R I}\right| \overrightarrow{0}, \overrightarrow{0}, \overrightarrow{0}>$, (b) $|\bar{\psi}>\otimes| \overrightarrow{0}, \vec{\alpha}, \overrightarrow{0}>$, and (c) $|\bar{\psi}>\otimes| \overrightarrow{0}, \overrightarrow{0}, \vec{\alpha}>$.

The $\mathbb{Z}_{2}$ symmetry in the heterotic string theory can be defined as a product of two $\mathbb{Z}_{2}$ 's, namely $\tau \otimes \sigma$, where $\tau$ is the inversion of the $(8,24)$ lattice and $\sigma$ exchanges the two $E_{8}$ lattice vectors. The momentum vectors defining the roots of the two $E_{8}$ 's are interchanged under the $\mathbb{Z}_{2}$ as $\left(\vec{k}, \overrightarrow{k_{1}}, \overrightarrow{k_{2}}\right) \rightarrow\left(-\vec{k},-\overrightarrow{k_{2}},-\overrightarrow{k_{1}}\right)$. 16-dimensional vector defining the internal coordinates of $\mathbb{T}^{8}$ changes sign under this operation, i.e., $x^{i} \rightarrow-x^{i}$ for $i=1, . ., 8$. Its operation on fermions can be found by decomposing them into a two-dimensional and an eight-dimensional part. They are given by the decomposition of $\mid \bar{\psi}^{+}>$as $\mid \psi^{+} \chi_{8}^{+}>$and $\mid \psi^{-} \chi_{8}^{-}>$, with $\mid \chi_{8}^{+}>\left(\mid \chi_{8}^{-}>\right)$being even (odd) under $\mathbb{Z}_{2}$.

Then the $\mathbb{Z}_{2}$-invariant bosonic states are the (a) graviton, (b) 64 scalars in (ii.a) above which are also singlets of the surviving nonabelian group $E_{8}$. In addition we have the states in the adjoint representation of the surviving $E_{8}$. They are the linear combinations of the states in (ii.b), (ii.c) and (ii.d) above and can be written as (c) $\psi_{-\frac{1}{2}}^{i} \mid 0>\otimes(|\overrightarrow{0}, \vec{\alpha}, \overrightarrow{0}>-| \overrightarrow{0}, \overrightarrow{0}, \overrightarrow{-\alpha}>)$, and (d) $\psi_{-\frac{1}{2}}^{i}\left|\overrightarrow{0}>\otimes X_{-1}^{+I}\right| \overrightarrow{0}, \vec{\alpha}, \overrightarrow{0}>$, where $X^{ \pm R I}$ is defined as $\left(X_{-1}^{(1) R I} \pm X_{-1}^{(2) R I}\right)$, with $X^{(1) R I}$ and $X^{(2) R I}$ denoting the coordinates in the Cartan subalgebra of the first and second $E_{8}$ respectively. The $\mathbb{Z}_{2}$ invariant fermionic 
states are the eight positive chirality gravitinos: (a) $\left|\psi^{+} \chi_{8}^{+}>\otimes X_{-1}^{\mu}\right| \overrightarrow{0}, \overrightarrow{0}, \overrightarrow{0}>$, (b) 64 spinors of negative chirality which are singlets of $E_{8} \times E_{8}:\left|\psi^{-} \chi_{8}^{-}>\otimes X_{-1}^{i}\right| \overrightarrow{0}, \overrightarrow{0}, \overrightarrow{0}>$, (c) 8 spinors of positive and 8 of negative chirality in the adjoint representation of $E_{8}$ namely: $\left|\psi^{+} \chi_{8}^{+}>\otimes(|\overrightarrow{0}, \vec{\alpha}, \overrightarrow{0}>+| \overrightarrow{0}, \overrightarrow{0},-\overrightarrow{-\alpha}>),\right| \psi^{-} \chi_{8}^{-}>\otimes(|\overrightarrow{0}, \vec{\alpha}, \overrightarrow{0}>-| \overrightarrow{0}, \overrightarrow{0},-\overrightarrow{-\alpha}>)$, $\left|\psi^{-} \chi_{8}^{-}>\otimes X_{-1}^{+R I}\right| \overrightarrow{0}, \overrightarrow{0}, \overrightarrow{0}>$ and $\left|\psi^{+} \chi_{8}^{+}>\otimes X_{-1}^{-R I}\right| \overrightarrow{0}, \overrightarrow{0}, \overrightarrow{0}>$.

The contributions of the spinors in the adjoint representation of $E_{8}$ cancel out in the anomaly computation as they come in pairs of opposite chirality. Since the spectrum in the twisted sector remains unchanged, the theory is anomaly free at the enhanced symmetry point. At generic points the full spectrum matches with the ones for the compactification of $M$-Theory found earlier in this section.

\section{Orientifolds of $K 3 \times K 3 \times \mathbb{S}^{1}$}

To obtain the two dimensional spectrum for this case, we now decompose the 11dimensional indices $M$ into $\mu, m, a$ and $x^{10}$, where $\mu, m$ and $a$ denote the two dimensional space-time, and the two $K 3^{\prime}$ 's: $K 3^{(1)}$ and $K 3^{(2)}$ respectively. Then the elevendimensional metric $G_{M N}$, upon compactification to two dimensions, gives among other fields, a graviton $G_{\mu \nu}, 58$ scalars $G_{m n}$ appearing as the moduli of the first $K 3$, another 58 scalars appearing as the moduli of the second $K 3$ and one more scalar, namely $G_{10,10}$. The ten-dimensional three-form field $A_{M N P}$ gives, upon compactification, 22 scalars $A_{m n 10}$ and another 22 scalars $A_{a b 10}$ coming from the two-forms on $K 3$ 's. In the physical bosonic spectrum we have 160 scalars in two dimensions.

The number of fermions can once again be counted by using the index of the Dirac and Rarita-Schwinger operators on both the K3's. The ten-dimensional spinor $\Psi_{\bar{\mu}}^{+}$ now gives the following set of fields: (i) 4 positive chirality spin- $\frac{3}{2}$ fields $\left(\psi_{\mu}^{+} \chi_{K_{1}}^{-} \chi_{K_{2}}^{-}\right)$, (ii) Two sets of 80 spinors of negative chirality: $\left(\psi^{-} \chi_{K_{1}}^{-} \chi_{K_{2} m}^{+}\right.$and $\left.\psi^{-} \chi_{K_{1} m}^{+} \chi_{K_{2}}^{-}\right)$. Similarly $\Psi_{\bar{\mu}}^{-}$contributes in two dimensions, four spin- $\frac{3}{2}$ fields of negative chirality and two sets of 80 spin- $\frac{1}{2}$ fields of positive chiralities. The ten dimensional spin- $\frac{1}{2}$ fields $\Psi_{10}^{+}$and $\Psi_{10}^{-}$contributes, in two dimensions, 4 spin- $\frac{1}{2}$ fields of positive and negative chiralities.

By cancelling the negative physical degrees of freedom of the gravitino with the 4 spin- $\frac{1}{2}$ fields of positive and 4 of negative chiralities, we are left with 160 spinors of positive and 160 of negative chiralities. By taking into account the scalars, we have a $(4,4)$ supersymmetric model with its 40 scalar multiplets as the matter content. The theory is non-chiral and anomaly-free. The orientifold of $K 3 \times K 3 \times \mathbb{S}^{1}$ can be constructed in the same way as in the previous sections, with the $\mathbb{Z}_{2}$ action on the two K3's defined in an identical fashion. This action can be summarized as $A_{M N P} \rightarrow-A_{M N P}, \psi_{M} \rightarrow \gamma^{11} \psi_{M}$ and $x^{10} \rightarrow-x^{10}$. Its action on individual $K 3$ 's is also described earlier.

Using these results we find that there are 96 scalar fields from the untwisted sector in this theory in the physical bosonic spectrum. The $\mathbb{Z}_{2}$ invariant fermion 
fields in two dimensions are as follows. The ten-dimensional spinor $\Psi_{\bar{\mu}}^{+}$gives the following $\mathbb{Z}_{2}$ invariant states in two dimensions (i) 4 spin- $\frac{3}{2}$ fields $\left(\psi_{\mu}^{+} \chi_{k_{1}}^{-} \chi_{k_{2}}^{-}\right)$(ii) Spin- $\frac{1}{2}$ fields $\psi^{-} \chi_{k_{1}}^{-} \chi_{k_{2} m}^{+}$and $\psi^{-} \chi_{k_{1} m}^{+} \chi_{k_{2}}^{-} . \Psi_{\bar{\mu}}^{-}$contributes $\psi^{+} \chi_{k_{1} m}^{+} \chi_{k_{2}}^{-}$and $\psi^{+} \chi_{k_{1}}^{-} \chi_{k_{2} m}^{+}$. These together give 4 spin- $\frac{3}{2}$ fields of positive chirality, 96 spin- $\frac{1}{2}$ fields of negative chirality and and 64 spin- $\frac{1}{2}$ fields of positive chirality. $\Psi_{10}^{-}$contributes only four negative chirality states $\left(\psi^{-} \chi_{k_{1}}^{-} \chi_{k_{2}}^{-}\right)$and $\Psi_{10}^{+}$does not have any $\mathbb{Z}_{2}$-invariant state. In this case we have 96 negative and 64 positive chirality spinors in two dimensions. Now we have a $(4,0)$ supersymmetric theory in two dimensions with the spectrum,

- $\left(1 g_{\mu \nu}, 1 \phi, 4 \psi_{\mu}^{+}, 4 \psi^{-}\right)$

- $\left(96 \phi_{L}, 96 \psi^{-}\right)$

- $\left(96 \phi_{R}, 64 \psi^{+}\right)$.

The left (negative)-chirality modes in the untwisted sector are combined into 24 multiplets of the $(4,0)$ theory. The right (positive)-chirality modes consist of 96 rightmoving scalars and $64 \mathrm{mw}$ spinors. The anomaly cancellation now requires $128 \mathrm{mw}$ spinors of positive chirality. It is noticed that this is exactly the number of the twisted sectors in this theory. Once again, taking into account the fact that the individual twisted sector contribution remains the same as for $\mathbb{T}^{9} / \mathbb{Z}_{2}$, we get cancellation of anomaly in two dimensions.

We now discuss the enhanced symmetry point on $\left(K 3 \times K 3 \times \mathbb{S}^{1}\right) / \mathbb{Z}_{2}$ orientifolds. Once again the approach is to find out the states in the heterotic string theory, identify the projections in these theories and obtain the invariant spectrum with respect to these projections. The $M$-Theory on $K 3 \times K 3 \times \mathbb{S}^{1}$ is equivalent to the heterotic string theory on $K 3 \times \mathbb{T}^{4}$. As a result the states of the former can be re-expressed as the states of the latter one. In this case, we obtain the two dimensional model by compactifying the heterotic string theory in two steps, first to six dimensions on $\mathbb{T}^{4}$ and then to two dimensions on $K 3$. By defining coordinates $X^{\tilde{\mu}},(\tilde{\mu}=0, \cdots, 5), X^{i}$ $\left(i \in \mathbb{T}^{4}\right), X^{R I}(I=1, \cdots, 16)$ as the bosonic worldsheet coordinates and $\psi^{L \tilde{\mu}}$ and $\psi^{L i}$ as the fermionic coordinates on the worldsheet we get the following set of states in this theory in six dimensions: (i) Graviton $\left(G_{\tilde{\mu} \tilde{\nu}}\right)$, antisymmetric tensor $\left(B_{\tilde{\mu} \tilde{\nu}}\right)$ and the dilaton $\phi$ arising as various combinations of the states: $\psi_{-\frac{1}{2}}^{L \tilde{\mu}}\left|0>\otimes X_{-1}^{R \tilde{\nu}}\right| \overrightarrow{0}, \overrightarrow{0}, \overrightarrow{0}>$, (ii) 16 scalars $\phi^{i j}$ which are also the singlets of $E_{8} \times E_{8}: \psi_{-\frac{1}{2}}^{L i}\left|0>\otimes X_{-1}^{R j}\right| \overrightarrow{0}, \overrightarrow{0}, \overrightarrow{0}>$, (iii) 4 scalars in the adjoint representation of $E_{8} \times E_{8}$ written as: (a) $\psi_{-\frac{1}{2}}^{L i}\left|0>\otimes X_{-1}^{R I}\right| \overrightarrow{0}, \overrightarrow{0}, \overrightarrow{0}>$, (b) $\psi_{-\frac{1}{2}}^{L i}|0>\otimes| \overrightarrow{0}, \vec{\alpha}, \overrightarrow{0}>$, and (c) $\psi_{-\frac{1}{2}}^{L i}|0>\otimes| \overrightarrow{0}, \overrightarrow{0}, \vec{\alpha}>$,

Upon further compactification to two dimensions on $K 3$ we obtain from $G_{\tilde{\mu} \tilde{\nu}}$, a graviton $G_{\mu \nu}$ and 58 scalars $G_{m n}$. $B_{\tilde{\mu} \tilde{\nu}}$ in six dimensions gives 22 scalars $B_{m n}$. In addition we also have the six dimensional scalars. Counting of the states leads to the bosonic spectrum of $M$-Theory at the enhanced symmetry point. This consists of the graviton, 97 scalars as singlets of $E_{8} \times E_{8}$ and 8 set of scalars in the adjoint of 
$E_{8} \times E_{8}$. At a generic point in the moduli space we have 161 scalars which matches with the ones presented earlier in this section for the $M$-Theory compactification.

The action of $\mathbb{Z}_{2}$ on the ten dimensional field as well as on $\mathbb{T}^{4}$ is defined exactly in the same manner as in the previous sections. Its action on $K 3$ is the same as the one mentioned earlier. The bosonic states of the heterotic string theory surviving the projection are the graviton $G_{\mu \nu}, \phi, 34$ scalars $G_{m n}, 14$ scalars $B_{m n}, 16$ scalars $\phi^{i j}$, all appearing as singlets of $E_{8}$. In addition we have 4 copies of scalars in the adjoint of $E_{8}$ appearing as the set of states: (i) $\psi_{-\frac{1}{2}}^{L i}\left|0>\otimes X_{-1}^{+R I}\right| \overrightarrow{0}, \overrightarrow{0}, \overrightarrow{0}>$ and (ii) $\psi_{-\frac{1}{2}}^{L i} \mid 0>\otimes(|\overrightarrow{0}, \vec{\alpha}, \overrightarrow{0}>-| \overrightarrow{0}, \overrightarrow{0}, \overrightarrow{-\alpha}>)$.

The fermionic spectrum is found by writing down the fermionic states in the heterotic language: (i) Gravitinos and the dilatinos in six dimensions appearing as the decomposition of the state: $\left|\bar{\psi}>\otimes X_{-1}^{R \tilde{\nu}}\right| \overrightarrow{0}, \overrightarrow{0}, \overrightarrow{0}>$ into six and four dimensional $\left(\mathbb{T}^{4}\right)$ parts. (ii) $E_{8} \times E_{8}$ singlet spinors: $\left|\bar{\psi}>\otimes X_{-1}^{R i}\right| \overrightarrow{0}, \overrightarrow{0}, \overrightarrow{0}>$ and (iii) spinors in the adjoint of $E_{8} \times E_{8}$ : (a) $\left|\bar{\psi}>\otimes X_{-1}^{R I}\right| \overrightarrow{0}, \overrightarrow{0}, \overrightarrow{0}>$, (b) $|\bar{\psi}>\otimes| \overrightarrow{0}, \vec{\alpha}, \overrightarrow{0}>$ and (c) $|\bar{\psi}>\otimes| \overrightarrow{0}, \overrightarrow{0}, \vec{\alpha}>$. Further compactification of these spinors to two dimensions is done in the same way as in sections-(2) and (3). For example, a state $\left|\bar{\psi}>\otimes X_{-1}^{R \tilde{\nu}}\right| \overrightarrow{0}, \overrightarrow{0}, \overrightarrow{0}>\equiv$ $\left|\bar{\psi}_{\tilde{\mu}}^{+}\right\rangle$is decomposed as a spin- $\frac{3}{2}$ states $\left|\psi_{\mu}^{-} \chi_{k}^{-} \chi_{4}^{+}\right\rangle,\left|\psi_{\mu}^{+} \chi_{k}^{-} \chi_{4}^{-}\right\rangle$, and spin- $\frac{1}{2}$ states $\left|\psi^{+} \chi_{k m}^{+} \chi_{4}^{+}\right\rangle,\left|\psi^{-} \chi_{k m}^{+} \chi_{4}^{-}\right\rangle$. By using the Dirac and Rarita-Schwinger index on $K 3$ we obtain 4 spin- $\frac{3}{2} \mathrm{mw}$ fermions: $\left(\left|\psi_{\mu}^{+} \chi_{k}^{-} \chi_{4}^{-}\right\rangle\right)$which are even under $\mathbb{Z}_{2}$. They also give us 32 positive and 48 negative chirality $\mathbb{Z}_{2}$-even spin- $\frac{1}{2}$ states and same number of odd ones. For other ten dimensional states, the decomposition of the $E_{8} \times E_{8}$ singlet states (ii) give 16 even states of negative chirality and 16 odd ones of positive chirality. For the spin- $\frac{1}{2} \mathbb{Z}_{2}$-even states which are in the adjoint of the $E_{8} \times E_{8}$ gauge group, we have 4 of them of positive and 4 of negative chirality. By counting all the even states we get 4 gravitinos appearing as positive chirality mw spinors. Among the spin- $\frac{1}{2}$ negative chirality states, we have 64 singlets of $E_{8}$ and 4 adjoints. Among the positive chirality ones are the 32 singlets and 4 adjoints of $E_{8}$. Since the adjoint representation appears as a pair of opposite chirality states, anomaly cancellation is maintained.

\section{Conclusions}

To conclude, we have presented $M$-Theory compactifications to two dimensions. We have shown that compactification on orientifolds leads to chiral theories in two dimensions. By taking into account the twisted sector states, anomaly cancellation is shown in many examples. The index theorem on $K 3$ and a $\mathbb{Z}_{2}$ abelian automorphism of these manifolds play an important role in the computations. The anomaly cancellation at some enhanced symmetry points have also been shown. Our results confirm the number of twisted sectors and their contributions as given in 5, 6. We have also shown that the states obtained are always in the representations of the unbroken supersymmetry algebra. 
In this paper we have been able to obtain $(16,0),(8,0)$ and $(4,0)$ models through the orientifold compactifications. It will be interesting to extend this analysis to obtain $(2,0)$ and $(1,0)$ chiral models in two dimensions. Considering the Calabi-Yau space for this purpose does not seem to change the number of supersymmetries, as

they have to be combined with the extra $\mathbb{T}^{3}$ leaving once again $\frac{1}{4}$ supersymmetry unbroken. Other compactifications such as on Joyce manifolds may have to be considered for this purpose. Other option may be to find other automorphisms of $K 3$ which break the supersymmetries further. It may also be interesting to analyze the moduli space of these theories and find out the Geroch group structure [26]. Normally the symmetries of the three dimensional theories are affinized on compactification to two dimensions. One, however, has to write down the effective action for the purpose of examining the Geroch symmetry structure and therefore it may be useful to understand these models in terms of the type IIA and type IIB string compactifications 13.

\section{References}

[1] E. Witten, "String Theory Dynamics in Various Dimensions", Nucl. Phys. B 443 (1995) 85.

[2] J.H.Schwarz, "The Power of M Theory", hep-th /9510086.

[3] J. Maharana, "M Theory and p-branes", hep-th /9511159.

[4] P. Horava and E. Witten, "Heterotic and Type String Dynamics from Eleven Dimensions", hep-th/9510209; "Eleven-Dimensional Supergravity on a Manifold with Boundary", hep-th/9603142.

[5] K. Dasgupta and S. Mukhi, "Orbifolds of M-Theory", hep-th /9512196.

[6] E. Witten, "Five-branes and M Theory on an Orbifold", hep-th /9512219.

[7] J.H. Schwarz, "M Theory Extensions of T-Duality", hep-th /9601077.

[8] K. Davis, "M-Theory and String-String Duality", hep-th/9601102.

[9] A. Sen, "M-Theory on $K 3 \times \mathbb{S}^{1} / Z_{2}$ ", hep-th /9602010.

[10] A. Kumar and K. Ray, "M-Theory on Orientifolds of $K 3 \times \mathbb{S}^{1} / \mathbb{Z}_{2}$ ", hepth/9602144 (To appear in Phys. Rev. D).

[11] O. Aharony, J. Sonnenschein and S. Yankielowicz, "Interactions of Strings and D-Branes from M theory", hep-th/9603009; O. Aharony, "String Theory Dualities from M Theory", hep-th/9604103. 
[12] B. Acharya, " $\mathrm{N}=1$ Heterotic/M-theory Duality and Joyce Manifolds", hepth/9603033.

[13] A. Sen, "Orbifolds of M-Theory and String Theory", hep-th/9603113.

[14] R. Dijkgraaf, E. Verlinde and H. Verlinde, "BPS Quantization of the FiveBrane", hep-th/9604052.

[15] S. Ferrara, R. Khuri and R. Minasian, "M-Theory on a Calabi-Yau Manifold", hep-th/9602102; S. Ferrara, R. Minnasian and A. Sagnotti, "Low Energy Analysis of $M$ and $F$ Theories on Calabi-Yau Threefolds", hep-th/9604097.

[16] F. Aldabe, "Heterotic and Type I Strings from Twisted Supermembranes" hepth/9603183;

[17] L. Alvarez-Gaumé and E. Witten, "Gravitational Anomalies", Nucl. Phys. B 234 (1984) 269.

[18] P. Townsend, Phys. Lett. B 139 (1984) 283.

[19] A. Dabholkar and J. Park, "An Orientifold of Type IIB Theory on K3", hepth/9602030.

[20] E. Gimon and C. Johnson, "K3 Orientifolds", hep-th/9604129.

[21] H. Nicolai and N. Warner, "The Structure of N=16 Supergravity in Two Dimensions", Commun. Math. Phys. 125, 369 (1989).

[22] A. Salam and E Sezgin, in Supergravities in Diverse Dimensions; NorthHolland/World Scientific, p 760.

[23] M. Walton, "Heterotic String on simplest Calabi-Yau manifold and its orbifold limits", Phys. Rev. D37 (1988) 377.

[24] S. Chaudhuri and D. Lowe, "Type IIA-Heterotic Duals with Maximal Supersymmetry", hep-th/9508144.

[25] E. Bergshoeff, H. Nishino and E. Sezgin, "(8, 0) Locally Supersymmetric Sigma Models with Conformal Invariance in Two Dimensions", Phys. Lett. B186, 167 (1987).

[26] A. Sen, "Duality Symmetry Group of Two Dimensional Heterotic String Theory", Nucl. Phys. B447, 62 (1995); A. Biswas, A. Kumar and K. Ray, "Symmetries of Heterotic String Theories", Nucl. Phys. B453, 181 (1995). 